\title{
Subitizing and counting depend on different attentional mechanisms: Evidence from visual enumeration in afterimages
}

\author{
TONY J. SIMON and SANDEEP VAISHNAVI \\ Georgia Institute of Technology, Atlanta, Georgia
}

\begin{abstract}
Two experiments showed that, when selective eye movements were disabled by the presentation of stimuli in the form of afterimages, increased inspection time and facilitative stimulus configurations failed to increase the subitizing limit of 4 objects. Afterimages of two to eight dots induced by a photographic flashgun were shown to 3 adult subjects. For more than 4 objects, enumeration errors occurred at a rate of $20 \%-30 \%$. Enumeration was effectively perfect for $2-4$ linearly configured dots, with occasional errors surprisingly occurring in that range when dots appeared in groups of up to 3 items. No errors occurred in nonafterimage control conditions. Enumeration errors were attributed to failures of individuating dots to be counted due to the deactivation of selective eye movements in afterimages. A third experiment supported this interpretation by disabling eye movements with briefly presented stimuli and producing results much like those of the afterimage conditions.
\end{abstract}

Recent attempts to explain visual enumeration phenomena have focused on the role of object individuation. Specifically, Trick and Pylyshyn (1993, 1994) have suggested that the characteristics of subitizing, or rapid, accurate visual enumeration of small sets of entities, are due to the process of assigning identification markers, known as FINSTs, to target objects. Subitizing is indicated by a performance profile in which 3 to 4 entities are enumerated at a rate of about $50 \mathrm{msec}$ per item with little or no error. This contrasts with a rate of around $300 \mathrm{msec}$ per item, along with numerous errors, for larger sets of objects, where a number of strategies, including counting, grouping, and estimating, may be employed. Such a profile is frequently interpreted as indicating some parallel component to object individuation in the subitizing range, while all processing for larger numerosities is thought to be serial.

That parallel/serial distinction has been explicitly made at both the theoretical and empirical levels. The results of Trick and Pylyshyn's (1993) series of experiments are consistent with their claim that FINSTs are assigned in parallel to a small number of target items during the preattentive phase of object recognition. The assumption that FINSTs are a limited resource provides Trick and Pylyshyn with an explanation of why the same

\footnotetext{
We would like to thank John Jonides and Steven Tipper for helpful comments on earlier drafts of this paper, and Zenon Pylyshyn and three anonymous reviewers for further helping us to improve the quality of the final product. We are also indebted to Beth Davis for her advice during the early stages of this research, and for her help in taking photometer readings. We would also like to thank members of our lab group for their input to this project. Correspondence regarding this manuscript should be addressed to T. J. Simon, School of Psychology, Georgia Institute of Technology, Atlanta, GA 30332-0170 (e-mail: tony.simon@psych.gatech.edu).
}

cannot be done for larger sets of objects, which must instead be individuated and then enumerated one at a time. Some neuropsychological evidence relevant to the serial/ parallel distinction between subitizing and counting has recently been reported by Dehaene and Cohen (1994). It concerns the enumeration performance of simultanagnosic patients, who can recognize and process single items but exhibit severely impaired serial visual exploration. According to Deheane and Cohen, this arises because simultanagnosics "fail to perceive the visual scene as a whole and report only some of its elements" (p. 960). This appears to signal an inability to serially apply the kind of object tags that are otherwise employed to individuate separate items in visual input. Such patients showed accurate enumeration for up to 3 items, which is within the subitizing range, but were unable to accurately enumerate larger sets, which would require stimulus items to be serially individuated. Although one should use caution in drawing inferences about the seriality or parallelism of processes underlying observable behavior (Townsend, 1990), these results do at least suggest that subitizing and counting are based upon quite distinct attentional processes.

In this study, we investigated that possibility further. However, instead of limiting the time resource, as is typical of subitizing studies, we manipulated the availability of eye movements as a mechanism of serial object individuation. Previous studies had tried to control serial scanning by either presenting stimuli too briefly to enable eye movements or requiring subjects to give highly speeded responses. In the latter design, different reaction time patterns were taken to indicate the deployment of different enumeration processes. Our idea, however, was to examine which enumeration range was most affected when one method of individuation, that of selective 
foveation, was rendered ineffective. This can be done while providing what would, under normal circumstances, be ample inspection time for fully accurate enumeration. Since it is thought to be counting and not subitizing which requires such a serial individuation mechanism, we predicted that our manipulation would only negatively affect performance in the larger number range. If removing the effectiveness of eye movements as a means of individuation negatively affects performance in the postsubitizing range but not the subitizing range, then our experiments would provide further support for the notion of distinct individuation processes underlying these different enumeration modes.

Therefore, in the following experiments, we controlled the deployment of eye movements as a means of selective individuation, first by the use of stimuli presented in the form of afterimages and second by presenting stimuli for exposure times too short to permit eye movements. In both cases, although subjects could move their eyes, they were robbed of the ability to selectively foveate individual items in the arrays. In the case of afterimages, this is because the entire image moves in concert with the eyes, whereas in the case of rapidly presented stimuli, the array has been removed and masked before more than a single eye movement can be completed.

Some precedent for such a manipulation does exist in the literature. Despite providing few details about how their experiment was done, Atkinson, Campbell, and Francis (1976) showed that adult subjects exhibited the familiar subitizing profile for enumerating dots that they could see for up to a minute when the stimuli were presented in the form of an afterimage. In other words, few if any errors were made in the $N \leq 4$ range while $20 \%$ to $30 \%$ errors were made for larger numerosities. Since the subjects had more than enough time in which they could ordinarily have counted the dots, their inability to enumerate any more than 4 dots without error suggests a limit to the number of items that could be accurately individuated without some form of deliberate attentional processing. This is because we must assume that these adults could count, and that the only reason that errors arose was that they could not accurately represent and individuate the to-be-counted objects. Atkinson et al.'s findings strongly suggest a limited representational capacity for items in the visual field when foveation is deactivated as an effective visual-search mechanism.

Of course, such a claim is hardly novel. The very construct of selective attention assumes a limited-capacity channel through which attended information can be encoded at relatively high levels of resolution, leaving other information to be processed cursorily, if at all. Within visual attention, metaphors such as spotlights (LaBerge, 1983 ) and zoom lenses (Eriksen \& St. James, 1986) have been used to describe the process of selecting target from background information at varying levels of specificity. While we do not here endorse any one of those metaphors, we will also assume that there is a limit to the capacity to pick up visual information (e.g., Hoyer, 1990) wherein a small set of identifiable entities are encoded and indi- viduated in some detail. Like the notion of a zoom lens, the actual capacity of that resource depends largely on the qualitative nature of the entities being attended. Trick and Pylyshyn (1993) have shown that when the targets of enumeration are not easily identified (such as 3 letter Os in a field of letter Qs), the familiar subitizing performance profile is replaced by a response pattern that resembles those typical of counting. This suggests that the scope of attentional pickup has had to be narrowed to the granularity of individual items in order to discriminate targets from distractors, thus forcing enumeration into a deliberate item-by-item mode.

Our first experiment had two goals. One was simply an attempt to replicate the Atkinson et al. result. In the current climate of theorizing about subitizing as an attentional phenomenon, it would seem to be important to establish the validity of this result. Experiment 1 is particularly important because it controls so many critical aspects of the visual enumeration task. Eye movements are rendered ineffective as an individuation strategy because movement of the eyes simply produces movement of the entire afterimage. Therefore, individuation by means of foveation is impossible. This leaves only the resources of the covert attentional system as a means of examining the image. Furthermore, all limitations on processing time are removed. Subitizing experiments typically either present all numerosities for a single, short duration (such as $150 \mathrm{msec}$ ) or place subjects in a speed/accuracy tradeoff situation where they are required to produce a response as fast as they can while attempting not to compromise accuracy. When afterimages are used, subjects are able to view the dots for up to $60 \mathrm{sec}$, more than enough time for any enumerative processing to take place. This means that performance should be limited only by the ability of the covert attentional system to enable the representation of the items to be enumerated. Finally, a control condition can be introduced where the same stimuli are viewed as a standard visual presentation in order to confirm that the reintroduction of overt attention in the form of eye movements restores performance to the total accuracy one would expect from adult enumerators.

The other goal of this experiment was to provide a different and converging method for testing hypotheses about the nature of individuation as the basis for the performance patterns observed in previous experiments on subitizing. As the preceding discussion stated, individuation within the subitizing range is believed to proceed in parallel, with very high accuracy, as long as the target stimuli are distinct. Therefore, given our hypothesis that presenting stimuli in afterimages limits overt but not covert attention, we would expect that individuation would not be negatively affected in this range, and thus enumeration should remain highly accurate. However, enumeration outside the subitizing range is assumed to be beyond the capacity of such reliable processes and depends in part on serial search. Without selective foveation, it is possible that individuation may not be perfectly accurate because the mechanism best suited for that purpose has been rendered ineffective. Since the subjects in 
this experiment were competent counters, we can infer that any errors in enumeration must be due to errors in individuation. Therefore, finding the typical pattern of subitizing performance when subjects have up to a minute to examine visual stimuli in an afterimage would suggest that individuation is indeed the basis of the phenomenon, and that it occurs in a very different fashion for small and large numbers of objects.

\section{EXPERIMENT 1}

\section{Method}

Subjects. Three subjects ( 2 male and 1 female), with normal or corrected-to-normal vision, participated in the experiment. The subjects were graduate students or faculty of the Georgia Institute of Technology's School of Psychology. Five other subjects (1 male and 4 female undergraduate students of the Georgia Institute of Technology with normal or corrected-to-normal vision) participated in the control condition. They received extra credit for introductory psychology classes in return for their participation.

Materials. The stimuli were horizontal linear arrays of whitefilled circles (each with a diameter of $2.4 \mathrm{~mm}$ ) on black rectangles ( $7.62 \mathrm{~cm}$ long and $5.08 \mathrm{~cm} \mathrm{high).} \mathrm{The} \mathrm{linear} \mathrm{arrays} \mathrm{were} \mathrm{centered}$ horizontally and vertically in the rectangle so that the center point of any stimulus was $3.33 \mathrm{~cm}$ from the vertical edges and $2.54 \mathrm{~cm}$ from the horizontal edges. Stimulus slides were created by printing from 2 to 8 white-filled circles on each black rectangle on overhead projector acetate film. This had the effect of making the dots into transparent spaces in a black field. The dots were $3 \mathrm{~mm}$ apart from each other. Figure la presents an example of an array from the subitizing range while Figure $1 \mathrm{~b}$ presents an example array from the postsubitizing range. Stimuli in the control condition consisted of the same white-filled circles printed on paper, causing the dots to appear as white circles on a black field. The stimuli in both conditions were generated using the MacDraw II graphics package on a Macintosh IIfx. The longest array was $4.22 \mathrm{~cm}$ long and, when viewed at a distance of $40.64 \mathrm{~cm}$, subtended a visual angle of $5.93^{\circ}$.

Procedure. The following double-blind procedure was used in the experiment. Each of the 7 numerosities was presented 5 times, based on a random ordering for the 35 trials in the experiment, which was generated by a secondary experimenter. Before each session, the second experimenter placed each of the five stimuli to be presented into individually labeled covers constructed from black card. The stimuli could not be seen, but a small tab protruded from the cover to enable easy removal of the stimulus slide. The stimuli in the covers were then handed to the primary experimenter. Before each trial in the darkened experimental room, the primary experimenter slid each stimulus slide directly from its cover into the flashgun's filter holder and then returned it to its cover at the end of the trial. At the end of the session, all five covers were returned to the secondary experimenter. In this way, the primary experimenter never knew how many dots were being presented during the experiment and hence could not assess the accuracy of the subjects' responses. The primary experimenter recorded the subject's responses for all 35 trials, and only after all the data were collected were the results given to the secondary experimenter to align the responses with the actual numerosities presented during the experiment.

The subjects were tested individually. There were a total of five trials per session so that each subject completed seven sessions lasting approximately $30 \mathrm{~min}$ each. The subject was brought into a room with the lights switched off. A Pritchard photometer (Model PR-198A) registered no measurement of ambient light under these conditions. The subject sat with his or her chin placed on a chinrest $40.64 \mathrm{~cm}$ from a flashgun (Vivitar $285 \mathrm{HV}$, Guide
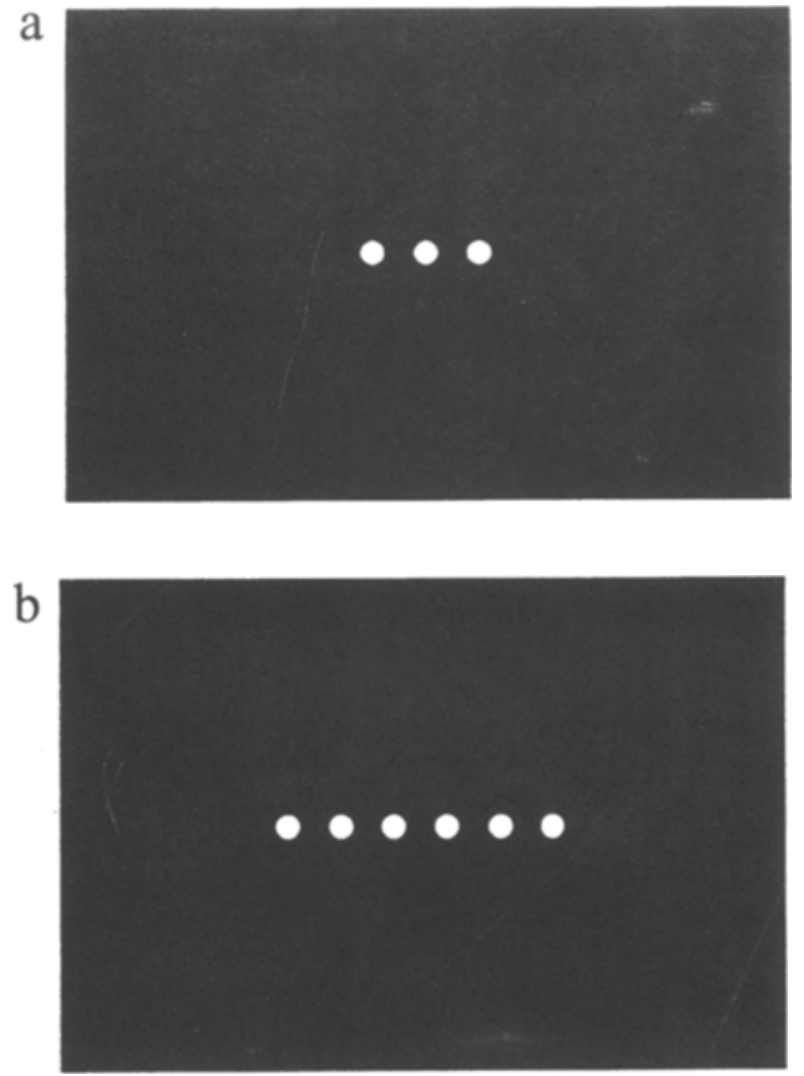

Figure 1. (a) Sample array from subitizing range of Experiment 1. (b) Sample array from postsubitizing range of Experiment 1 .

No. 100, wide angle, 100ASA) and $76.2 \mathrm{~cm}$ from a CRT (a highresolution 13-in. RGB Macintosh color display Model M1212), which emitted $9.05 \mathrm{~cd} / \mathrm{m}^{2}$ of light from a white screen. The subject was given $3 \mathrm{~min}$ to adapt to the low-light conditions.

Each trial consisted of the following steps: The CRT screen had a white background with the word "READY" in black capital letters $3 \mathrm{~cm}$ from the bottom of the screen (with a height of $5 \mathrm{~mm}$ and a length of $4 \mathrm{~mm}$ ). The subject was asked to look at the word "READY." The stimulus slide was placed in the flashgun filter holder and covered with a white piece of paper. The experimenter verbally counted down from 3 , and at the count of 1 , the subject was asked to look at the covered stimulus. The experimenter then simultaneously dropped the covering from the stimulus and fired the flash gun. The subject made two responses as to the number of dots that he or she saw in the afterimage and the confidence he/she had in that enumeration (with 1 being the lowest and 5 being the highest). The first response was made $10 \mathrm{sec}$ after the flashgun was fired, and the second was made after $60 \mathrm{sec}$. The subject was signaled to make a response by a tone emitted by the computer. The time intervals were controlled by the software package Cedrus Superlab running on a Macintosh IIfx. There was a 5-min break after each trial, during which the subject played a visually stimulating video game ("Tetris") on the computer. This interval, based on previous studies (e.g., Gerrits, van Erning, \& Eijkman, 1988), was designed to allow for the dissipation of any residual afterimage from the last trial. If the subject felt that he/she needed more time, an extra minute was given.

In the control condition, four changes were made. First, the stimuli were printed on paper instead of acetates, and so appeared as white circles on black backgrounds. No change was made to 
the means of presentation, since the stimuli were displayed in the flashgun's filter holder as before. Second, the room was fully lit and no afterimage was induced; instead, subjects simply enumerated the dots on the paper. Third, all 35 trials were completed in a single 30 -min session, since no afterimage dissipation periods were necessary. Finally, responses were given after 10 and after $30 \mathrm{sec}$ along with the confidence judgments. The 3 subjects participated in the control condition in a separate session after completing all 35 trials of the afterimage task. Five additional subjects who had not participated in the afterimage task also completed the 35 control-condition trials.

\section{Results}

To simplify description of the results and allow for maximal comparison with previous findings, we shall refer to the numerosities $N=2-4$ as the subitizing range and the numerosities $N=5-8$ as the postsubitizing range. We took this step for the following reasons. First, as Trick and Pylyshyn's (1994) meta-analysis shows, the overwhelming majority of studies indicate a subitizing span of 4 items despite employing a range of methodologies. While these are idealized estimates because they aggregate over individual differences, a strong consensus for a subitizing span of 4 items now exists. Second, the nature of the data produced by our experiments meant that the standard method of estimating subitizing span could not be used. Despite considerable effort, we were unable to find another appropriate exploratory analysis that would empirically derive a subitizing span. Thus, we will assume that subitizing has a span of up to 4 objects and will comment on exceptions when they occur.

Overall, the results in this experiment showed that subjects were highly accurate and highly confident in their enumerations within the subitizing range. They were less accurate and less confident in the postsubitizing range. All 3 subjects' data were aggregated together, and the pattern of results is presented in Figure 2. As can be seen from that figure, the mean percentage of error patterns, including standard errors, show quite distinct patterns within and outside the subitizing range.

There were $2.2 \%$ errors in the subitizing range for the 10 -sec response and no errors for the 60 -sec response. Standard error values for individual numerosities ranged from $0 \%$ to $5 \%$ in this range. The errors for the postsubitizing range were $18.35 \%$ and $15 \%$. Here, standard error values ranged from $0 \%$ to $14 \%$. Examination of Figure 2 shows that the difference was even more striking than these percentages describe. This is because the average values for the postsubitizing range were lowered by the increased accuracy and confidence for the largest numerosity. This is a rather standard finding, sometimes referred to as an "end effect." When subjects know the largest number of objects that can be presented, they often guess that quantity when presented with a dense display that they are unsure they can enumerate. As can be seen, errors for the larger-number stimuli were typically in the $20 \%$ to $30 \%$ range. Since chance responding is 1 in 7 , or $14 \%$, these results suggest that enumeration performance was above chance in the subitizing range but below chance for larger numerosities. Patterns simi-

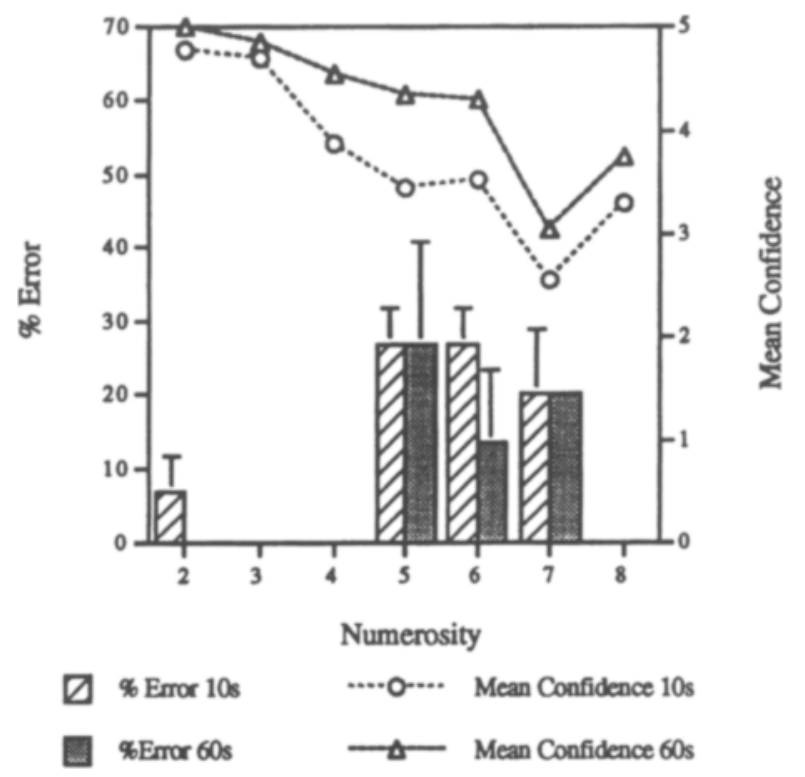

Figure 2. Aggregate accuracy and confidence data for Experiment 1.

lar to the accuracy data were evident in the mean confidence ratings. In the subitizing range, they were 4.44 and 4.79 out of 5 for the 10 - and 60 -sec responses. In the postsubitizing range, the ratings were 3.2 and 3.86 .

However, despite the dissimilarity in error rates between the small-number and larger number ranges, there remains the possibility that the differences are not significant. While performance in the small-number range was effectively error free, the majority of responses in the larger number range were also correct. The two patterns of errors may be noticeable but not statistically distinct. To test this hypothesis, we examined the probability that the pattern of errors in the subitizing range could have occurred by chance on the basis of the error rates in the postsubitizing range. This was done using tests of the binomial distribution, since our data did not conform to a normal distribution. First we took the average error rate for the numerosities 5 through 7 for the 10- and 60 -sec responses. The results for 8 objects were not included because responses to this numerosity were biased by the end effect. This resulted in the unique case of no errors being made for a large numerosity due to this guessing strategy. For the 10 -sec response, the aggregate error rate for 5-7 objects was $25 \%$. Using the binomial distribution, we tested for the probability that a zero-error pattern in the subitizing range could have occurred by chance on the basis of the postsubitizing error rate. This tests the probability that for the 15 responses given to each of the 3 numerosities concerned, none would be an error if the same processes responsible for postsubitizing performance were at work. Zero errors seemed the fairest value to test for since only a single error was made in all the 45 responses aggregated in this range. The probability of zero errors for $P=.25, N=15$ was $p=$ 
.013 , enough to strongly reject the null hypothesis of no differences between the subitizing and postsubitizing ranges. Similarly, taking the slightly lower rate of $20 \%$ errors for the postsubitizing 60 -sec response, the zeroerror pattern of the subitizing range was also very unlikely to have occurred by chance, $P=.2, N=15$ was $p=.035$. Thus, it seems safe to conclude that the pattern of errors outside the subitizing range was significantly higher than that inside the subitizing range.

In the control condition, none of the 3 subjects made any errors at all, showing perfect enumeration by the 10 sec response. This result cannot be attributed to practice on the task from the afterimage portion of the experiment because the 5 further subjects who did not experience the afterimage task produced exactly the same results.

\section{Discussion}

Overall, performance was slightly better than in the Atkinson, Campbell, and Francis (1976) study in that fewer errors were made in the $N \geq 5$ range. Also, the results of the original study were largely replicated. Enumeration within the subitizing range was effectively error free, while small numbers of errors were consistently made outside the subitizing range, even though counting 8 objects would ordinarily be a trivial task. The extra time available for inspection of the afterimage between the 10 - and $60-\mathrm{sec}$ response apparently had little or no effect on enumeration accuracy. This was despite subjects' reports that they did continue to try to examine the items in the afterimage for the entire $60 \mathrm{sec}$, especially in the case of the postsubitizing numerosities. Subjects' increased confidence ratings between their initial and delayed response, especially in the $N=4-6$ range, indicates that they believed the extra time was beneficial. However, it apparently had little effect on performance.

The fact that subjects produced totally accurate enumerations for all numerosities in the control condition not only shows the unsurprising result that they could count, but also suggests that they could enumerate accurately only when their overt attention system enabled a clear individuation of the objects to be counted. That was true for all numerosities in the control condition, where eye movements were effective, but only for up to 4 objects in the afterimage condition, where covert attention was the only resource.

Apparently, individuation of the items in the small numerosities occurred almost without error, enabling accurate enumerations to be made. Quite likely, this was done in parallel, as suggested by Trick and Pylyshyn (1993). Mistakes in enumeration in the larger ranges, where objects are identified one at a time, must be attributed to individuation failures. Either some items were missed and not counted or others were counted more than once. Such enumerations therefore violate what Gelman and Gallistel (1978) called the "one-one" principle of counting, which "involves the ticking off of items in an array with distinct tags ... in such a way that one and only one tick is used for each item in the array" (p. 77). This is a principle that young children must master on their way to accurate counting, and one that adult counters certainly know and use under normal circumstances. Why then were they unable to consistently do so here?

The data suggest that disabling selective foveation by presenting the stimuli in the form of afterimages was responsible for introducing error into the individuation mechanism. Errors were made on only a minority of trials in the postsubitizing range, but the data show a consistent pattern of misindividuation that occurred on at least 1 in 5 trials. Therefore, performance was worse than the chance level of $14 \%$ errors. No such errors were ever made in the control condition. Assuming parallel individuation within the subitizing range and serial individuation beyond it, many more attentional shifts would be required to move between individual dots outside the subitizing range. Therefore, we hypothesize that enumeration in this range may suffer due to the increased chance that one such shift might go awry. This would result in the missing or revisiting of an item. Under this interpretation, the data from Experiment 1 suggest that, when stripped of the ability to use eye movements as a means of selectively individuating stimulus items in the afterimage condition, subjects' attention apparently "got lost" on some trials involving serial individuation. This resulted in an inaccurate enumeration of the number of dots present in the array. According to Frick (1987), two components of counting are "ordering the objects linearly, such that each object is counted exactly once" and "objects have to be isolated and identified as individual units" (p. 8). Presumably, somewhere in that isolation and ordering process, the afterimage condition caused occasional failures to occur.

Therefore, one question that arose from this interpretation was whether all such shifts of attention must be carried out by using eye movements if they are to be totally accurate. It is possible that the uniformity of the stimuli in Experiment 1 made any method of shifting attention prone to error. Accordingly, Experiment 2 was designed to both reduce the number of attentional shifts required in the postsubitizing range and to make the resulting location of each shift easier to identify. Our aim was to see if the percentage of errors dropped as a result. The reasoning was that the fewer shifts the subject had to make and the clearer the result of each shift was, the less chance there was for attention to get lost and for individuation errors to be made. Thus, stimuli were grouped to thereby remove what Frick (1987) called the "homogeneity effect," where identical items in continuous rows are harder to count than identical items in "moderately disorganized" configurations. We expected that the manipulation would have no effect on performance in the subitizing range, where it is believed that no attentional shifts are made during individuation, thereby removing the likelihood of enumeration errors. 


\section{EXPERIMENT 2}

Our aim here was to organize stimulus objects into distinct groups, each with a number of members within the subitizing range. Given a field of a relatively large number of items, van Oeffelen and Vos (1982) showed that subjects will segment the array into small clusters of objects. Furthermore, Shrager, Klahr, and Chase (1982) showed that such organization produces an efficient enumeration strategy in adults, which they labeled as "tallying." Subjects segment the array into groups, preferably with two or three members, which can easily be subitized and whose quantity can be added to a running total. Therefore, we arranged our stimuli in this fashion so that the arrays afforded the use of the efficient tallying strategy. Also, no more than two shifts of attention between groups would ever be required because there was a maximum of three groups, each containing a subitizable number of dots. This, it was expected, would reduce the chances of attention "getting lost" when compared with the linear arrays, where up to seven shifts might be required.

\section{Method}

Subjects. The 3 subjects used in the first afterimage experiment participated in this experiment. Five additional undergraduate students (again, 4 females and 1 male with normal or correctedto-normal vision) were recruited to complete the control condition alone.

Materials. The stimulus objects were the same circles as those used in the first experiment except that they were no longer arranged in linear horizontal arrays. Instead, the dots were arranged into perceptually separable groups. Figure 3 a presents an example of an array from the subitizing range; Figure $3 \mathrm{~b}$ presents an example array from the postsubitizing range. No group ever had more than 3 dots, and there were three different patterns of groupings for each numerosity. On any given trial, the pattern for each numerosity was randomly chosen from the set of three. This guarded against subjects' learning to recognize numerosities from the pattern alone. The largest stimulus pattern covered an area of $1.19 \times$ $3.02 \mathrm{~cm}$ and subtended a visual angle of $4.48^{\circ}$.

Procedure. The procedure was exactly the same as that used in the first experiment

\section{Results}

Results from this experiment showed a rather different pattern from those in the first. Although subjects were more accurate and more confident in their enumerations within the subitizing range than in the postsubitizing range (as defined earlier), the difference was blurred considerably. Surprisingly, an occasional error was made within the subitizing range, and yet subjects seemed unaware of this, because their confidence ratings were little changed from those in the first experiment. However, error rates in the subitizing range remained below the chance rate of $14 \%$. Again, all 3 subjects' data were aggregated together, and the pattern of results is presented in Figure 4. The figure shows that, despite a small elevation of errors, 2- and 3-dot arrays produced the most accurate enumerations, while the 5 through 7 range produced rather reduced accuracy. Again, there is some evidence of an end effect for the $N=8$ displays. The slightly
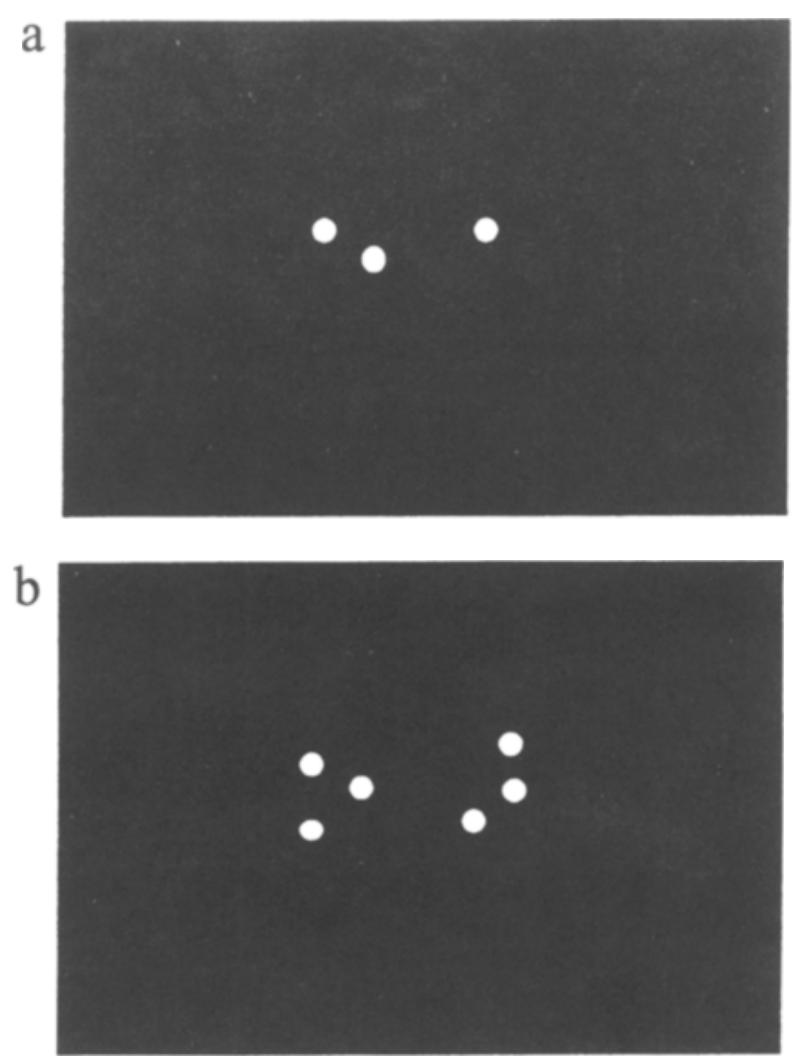

Figure 3. (a) Sample array from subitizing range of Experiment 2. (b) Sample array from postsubitizing range of Experiment 2.

unclear status of the $N=4$ data reflected some previous authors' ambivalence about which process was being used to enumerate such a set size (e.g., Svenson \& Sjöberg, 1983). In terms of accuracy, the responses to $4 \mathrm{ob}-$ jects were consistent with the above-chance responding associated with subitizing in Experiment 1, although the slightly elevated error rate seems to more closely resemble that of the larger numerosities.

There were $6.67 \%$ errors in the subitizing range for the 10 -sec response and $8.89 \%$ errors for the 60 -sec response. Standard-error values ranged between $0 \%$ and $10 \%$. In the postsubitizing range, the errors for the 10 - and 60 -sec responses were $21.67 \%$ and $10 \%$, respectively. Here standard error values ranged from $0 \%$ to $9 \%$. Again, aggregating over the postsubitizing values somewhat reduces the real differences in performance. Although Figure 4 shows a varied pattern of errors, only when subjects were enumerating 5 objects or more did errors reach into the $20 \%-30 \%$ range. In the subitizing range, mean confidence ratings were 4.28 and 4.71 out of 5 for the 10 and $60-\mathrm{sec}$ responses. In the postsubitizing range, they were 3.52 and 4.24 , respectively. Overall, then, confidence was higher in this second experiment, while accuracy was the same or marginally lower. This suggests that subjects thought they could enumerate the grouped stimuli more easily than the linearly arranged dots when in fact this was not the case. 


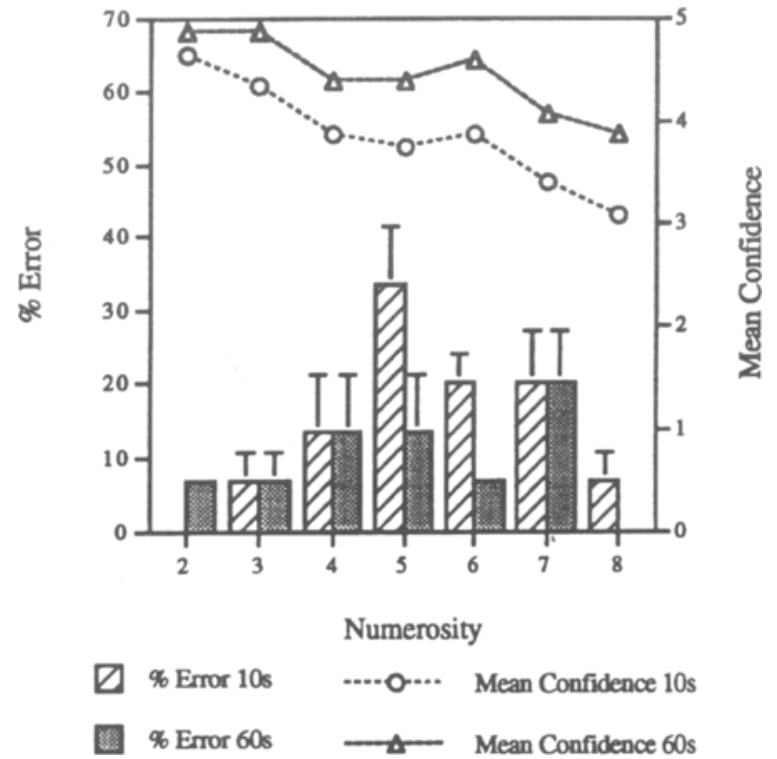

Figure 4. Aggregate accuracy and confidence data for Experiment 2.

Using the same reasoning as in Experiment 1, the performance of subjects in the subitizing range was compared with their performance in the postsubitizing range for the numerosities 5-7, using the binomial distribution test. For the 10-sec response, the null hypothesis of no differences could not be rejected. The error rate for $N=$ $5-7$ was $20 \%$. Since at least 1 error was made on average in the subitizing range, the combined probability of 0 errors $(P=.20, N=15, p=.035)$ and 1 error $(P=$ $.20, N=15, p=.132$ ) was .167 , suggesting that performance in the two ranges was not significantly different. For the 60 -sec response, the average error rate for $N=$ $5-7$ was $11 \%$, so the probabilities of $0(P=.10, N=15$, $p=.206)$ or $1(P=.10, N=15, p=.343)$ errors arising in the subitizing range was even higher at $p=.549$. Thus, because it did not confer the subitizing range advantage that the linear configuration did, it seems clear that the grouped condition actually made performance worse.

Again, in the control condition, no subject made any errors at all, showing perfect enumeration by the $10-\mathrm{sec}$ response. As in Experiment 1 , this result cannot be attributed to practice on the task from the afterimage portion of the experiment, because the 5 additional subjects, who did not experience the afterimage task, produced exactly the same results.

\section{Discussion}

We were initially surprised to find that the grouped stimuli did not confer an accuracy advantage over the linear configurations. In fact, performance on these stimuli was the same or slightly worse than that for the linear configurations. The extra time available between the 10 - and $60-\mathrm{sec}$ responses seemed, in this experiment, to have some facilitating effect on accuracy, at least in the postsubitizing range. This suggests that the subjects were able to exert some kind of control over search of the items in the afterimage. However, given the relatively consistent appearance of errors, even for the 60-sec responses, it is clear that this delivered only a small improvement in performance over their initially less accurate enumerations. The subjects still could not repeat the perfect accuracy of the control condition. Apparently, they had as much trouble individuating the items in the arrays designed specifically to facilitate that process; indeed, they did not perform any better in the postsubitizing range than they had in the first experiment. Even more surprising is that, although the actual frequency was very low, some errors were apparent even in the subitizing range. This, too, differs from the findings of Experiment 1.

It may be that some attentional shifts were induced by the small-number arrays' having distinct groups, thus occasionally producing errors. The stimuli in this experiment subtended around $4.5^{\circ}$ of visual angle. That is too large for foveal presentation of the entire array. Thus, under normal circumstances, any attempt at visual search would require eye movements. As Trick and Pylyshyn (1993) showed, arrays subtending visual angles of this size or greater do not interfere with subitizing as long as deliberate search is not necessary. Therefore, it seems likely that our subjects did occasionally engage in some attentional shifting between subgroups in these arrays. In other words, they may have attempted to use a shift of foveation to examine other parts of the stimulus array, which in the afterimage, but not the control, condition would have proved ineffective. Overall, though, we cannot tell whether subjects were unable to move accurately from one group to another or to accurately decompose each group; in any event, the grouping manipulation did not increase accuracy. It is interesting to note that, overall, subjects' confidence scores were slightly higher in this version of the task than when the stimuli were in linear arrays. This suggests, first, that the stimulus groupings were detected, and second, that subjects felt the task was easier when the stimuli were organized that way. Surprisingly, their performance did not reflect this high confidence. Subjects apparently felt confident that the groupings made enumeration easier and that visual scanning was being effective, yet, all the while, they were being failed by some aspect of the individuation process. This result suggests that selective foveation does act as a kind of control mechanism for navigating visual displays. It was necessary for the individuation of complex displays, and also it seemed to be required in order to exploit facilitative groupings that made enumeration easy when eye movements could be deployed.

\section{EXPERIMENT 3}

The experiments reported above both show that subjects make enumeration errors for arrays of more than 4 objects and, under some circumstances, when those objects are presented in afterimages, for smaller arrays. 
However, they never make any such mistakes when the same stimuli are presented in the form of more normal visual images. One major difference between these two forms of stimulus presentation is that the afterimage condition does not allow for the resulting visual representation to be searched, item by item, with the use of eye movements, whereas the control condition does. Thus, we have claimed that, when objects can be enumerated without eye movements (i.e., in the subitizing range of Experiment 1), performance is effectively error free. However, when eye movements are required or used voluntarily to select individual stimulus items, performance deteriorates to below-chance levels. We believe this is the basis for the difference between the control conditions and the afterimage conditions in the postsubitizing range of both experiments, and may explain the increased incidence of errors in the subitizing range of Experiment 2. Of course, this explanation is confounded with other possible explanations that relate to the special characteristics of the afterimage presentation. These might include factors such as the apparent and rather unpredictable motion of the dots experienced by subjects in the afterimage conditions. Therefore, we needed to test our eye-movement hypothesis directly by removing the ability of subjects to use eye movements for object individuation without presenting the stimuli in the form of an afterimage. If this experiment produced results like those of the afterimage condition, it would support our claim that it was the inability of subjects to individuate stimulus items by means of eye movements in the afterimage condition that was responsible for the appearance of errors, presumably arising from misindividuation.

\section{Method}

Subjects. The 3 subjects used in Experiments 1 and 2 took part in this experiment.

Materials. The stimuli were the same ones as those used in Experiments 1 and 2 , only now they were presented on a computer monitor screen. The stimuli were produced by the original graphics files used to produce the earlier stimuli. They had been saved in the form of PICT files and were presented by the same Superlab software used to control the timing in Experiments 1 and 2.

Procedure. Exactly the same number of trials (five for each of the seven numerosities) was presented for each of the two conditions in this experiment. In the linear condition, the stimuli for Experiment 1 were presented; in the grouped condition, the stimuli from Experiment 2 were presented. In both cases, presentation time for each trial was $100 \mathrm{msec}$. Stimulus configurations were sets of white dots in black rectangles, just as before. Each was preceded for 1 sec by a white fixation rectangle with a black border, and each was followed by a checkerboard mask made up of the same rectangle used for the stimuli but now filled with a dense checkerboard pattern. Once the mask appeared, the subjects were able to type the number of dots they thought had just been presented. The 35 trials were presented in randomized order, computed for each subject by the Superlab program.

\section{Results}

As can be seen in Figure 5, the results were rather similar to those in the afterimage conditions. However, one noticeable difference is that error rates are higher in the

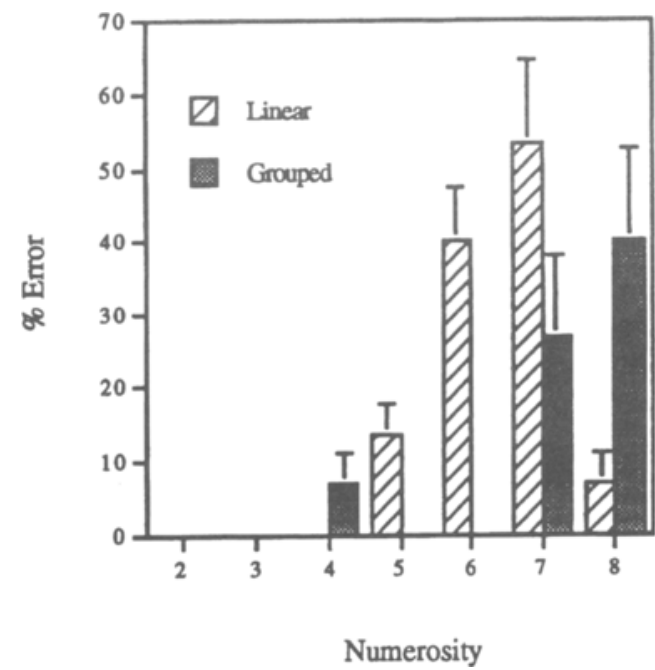

Figure 5. Aggregate accuracy data for Experiment 3.

postsubitizing range. This is almost certainly due to the very short presentation time used to ensure that eye movements could not be used. Some subitizing experiments present stimuli for a fixed exposure, typically 150-200 msec (e.g., Atkinson, Campbell, \& Fancis, 1976; Mandler \& Shebo, 1982), and they also report error rates of $20 \%-60 \%$ in the $4-8$ item range.

For the linear condition, once again no errors were made in the subitizing range of 2-4 items, while errors occurred at a slightly higher rate in the postsubitizing range than in the afterimage version of the task. The $0 \%$ errors in the subitizing range contrasted with $28.33 \%$ errors for the postsubitizing range, where standard error values ranged from $9.43 \%$ to $24.94 \%$ for individual numerosities. We tested for differences between these ranges, using the binomial distribution test in the same way as in Experiments 1 and 2. The aggregate error rate for $N=5-7$ was $35.55 \%$, and the probability of zero errors in the subitizing range based on that rate $(P=.35$, $N=15$ ) was $p=.002$. Therefore, it seems safe to conclude that subjects' performance was significantly worse outside the subitizing range than within it. This result replicates the findings of the afterimage condition in Experiment 1.

For the grouped condition, the error pattern was slightly different. Subjects barely made any mistakes in enumerating the grouped stimuli until there were 7 items presented. This is more like the pattern we were expecting in Experiment 2 and suggests that, in general, grouped stimuli are easier to enumerate because up to two groups of 3 items could have been easily enumerated within a fixation or two. This finding lends some support to our speculation that presenting the same stimuli for up to $1 \mathrm{~min}$ in afterimage form did, in fact, induce eye movements that were ineffective for individuation and hence served only to reduce accuracy under such conditions. Overall, the error rate for a subitizing range of $2-4$ ob- 
jects was $2.2 \%$. This drops to $1.3 \%$ if we alter the subitizing estimate to 6 objects, as the graph suggests. The standard error value was $4 \%$ for the single numerosity that produced an error. There were also slightly fewer errors in the postsubitizing range than in the afterimage condition of Experiment 2, but the rate was still $30 \%$ to $40 \%$ for the numerosities of 7 and 8 . The overall rate was $16.67 \%$, using a span of $N=5-8$. This increases to $33.3 \%$ if we adjust the postsubitizing span to $N=7-8$. Standard errors ranged from $0 \%$ to $13 \%$. Given the obvious lack of an end effect for these data, and sticking with the original estimate of subitizing, the binomial distribution test compared the error rate for numerosities 5-8 with that for numerosities 2-4. The rate, as stated, was $16.67 \%$, and, as in Experiment 1, a single error from 45 responses in the subitizing range was considered to be an error rate of $0 \%$. If we use the strict test of a $15 \%$ postsubitizing error rate, we again see that the pattern of errors seen outside the subitizing was unlikely to have occurred by chance $(P=.15, N=20, p=.012)$. This probability is a great deal lower than the Experiment 2 finding that performance in the subitizing range was not significantly better than performance in the postsubitizing range. Of course, this condition is one where the assumed estimate of subitizing span does not seem to fit so well. As in other, nonafterimage, experiments, the configural characteristics of the stimuli did appear to make enumeration of larger sets easier. Therefore, it seems valid to repeat the above analysis with the assumption that the grouped dots in this experiment increased the subitizing span to 6 objects, as suggested by examination of Figure 5 . If we compare the error rate of $0 \%$ with that of a conservative $30 \%$ for the adjusted postsubitizing range of $N=7-8$, we find further evidence that the increased rate was unlikely to have occurred by chance $(P=.30, N=10, p=.028)$.

\section{Discussion}

The results of both conditions in this control experiment support our interpretation of the differences between postsubitizing performance in the control and afterimage conditions of Experiments 1 and 2. In the linear condition, the result is very clear; in the grouped condition, it is marginally so. The latter result is consistent with the fact that easily decomposable stimulus configurations should improve accuracy beyond the subitizing range. Overall, though, the results suggest that it was the inability to individuate items in the arrays using selective eye movements that created up to $33 \%$ errors in the afterimage conditions. While other factors may have contributed, this experiment suggests that it is unlikely that the errors were due exclusively to some other aspect of the visual representation that was created under these unusual circumstances, such as the apparent movement of the stimulus configurations. Therefore, it seems that enumerations of small quantities of objects can proceed perfectly accurately without the need for selective eye movements as an individuation mechanism. Larger num- bers of objects appear to require some kind of pointing process for objects to be isolated and ordered for enumeration with perfect accuracy. In the case of the current experiments, that would seem to involve pointing with the eyes.

\section{GENERAL DISCUSSION}

In Experiments 1 and 2, subjects consistently made occasional enumeration errors outside the subitizing range when the stimuli were presented in the form of afterimages. No errors occurred in the control conditions, in which the same stimuli were presented in the form of standard visual images. This was true whether the stimuli were arranged in linear or in grouped configurations. One major difference between the afterimage and control conditions is that stimulus configurations presented in the form of afterimages cannot be selectively scanned by using eye movements, whereas normal visual stimuli can. This suggests that quantities within the subitizing range can be enumerated with periect accuracy without the need for some deliberate pointing mechanism, whereas larger quantities cannot. A third experiment, which rendered eye movements ineffective for individuation by presenting stimuli from Experiments 1 and 2 for very short durations, produced results very similar to those of the afterimage conditions. This supports our interpretation that the key difference between subitizing and postsubitizing performance is that the latter requires the use of eye movements as an individuation mechanism if perfect accuracy is to be achieved, while the former does not. Surprisingly, we found that varying the stimulus configurations from ones that are harder to ones that are easier to count (Frick, 1987) had no facilitative effect on postsubitizing accuracy in afterimage presentations. The fact that this occurred only when selective foveation was disabled provides further support for the idea that overt attentional mechanisms are required for fully accurate individuation, and thus for enumeration, of numerous displays.

The findings from the above experiments seem to make four important points. The first is that we have provided new and converging evidence that subitizing and counting arise when subjects deploy quite different attentional mechanisms. Our most striking result was the difference between enumeration performance in the postsubitizing range between the control and all experimental conditions. In the control conditions, subjects could use selective foveation and not a single error was made. In the experimental conditions, selective eye movements were rendered ineffective by afterimage presentation or impossible by brief presentation. In each case, the subjects who had performed without error in the control conditions now made $20 \%-30 \%$ enumeration errors. This suggests that serial individuation of objects, as occurs in counting, relies on selective eye movements to attain complete accuracy. In contrast, two experiments out of three found that disabling selective eye move- 
ments had no such deleterious effects on enumeration in the subitizing range. This supports the claim that the subitizing process arises from the operation of covert, and probably, preattentive individuation mechanisms. Only in the case where we suspect that subjects may have been induced to make unnecessary eye movements were a small number of errors evident. (For two of those three numerosities, performance was still above chance responding level.) Therefore, our results strengthen the link between subitizing and preattentive processing presented by Trick and Pylyshyn (1993, 1994).

A second, and related, implication of the results is that they strengthen the case for individuation processes being the basis of the subitizing phenomenon. Given that our subjects were competent counters, we must assume that errors in enumeration could only be due to errors in individuation. The current consensus is that the individuation of small numbers of objects occurs either in parallel or at least without the need for attentive search. Our findings of effectively error-free enumeration in the subitizing range, with or without selective eye movements, supports the view that items in this range can be processed and tagged without the need for explicit pointing. However, when the number of objects exceeded the subitizing range and overt serial search in the form of eye movements was prevented, enumeration errors were made. This suggests that unless serial search via foveation takes place, errors can occur in the process of determining which items have been counted and which ones have not. Our results also suggest that the same holds for determining which subgroups of a larger array have been subitized and which have not.

The third, and perhaps most novel, finding is that the subitizing limit, commonly accepted to be in the range of 4 objects, does not increase with greater inspection time as long as subjects cannot use eye movements to selectively attend to stimulus objects or groups. Commonly, subitizing experiments present stimuli for very brief durations and subjects are able to enumerate only around 4 objects quickly and accurately. This result was replicated in the linear condition of Experiment 3 (and in the grouped condition, the configurations made the task even easier). However, in Experiments 1 and 2, subjects in the afterimage conditions had access to the stimuli for up to a minute and were still unable to enumerate more than 4 dots with perfect accuracy. Therefore, extra inspection time does not increase the subitizing range of errorless enumeration unless eye movements can be used to mark off items that have been processed. As Frick (1987) succinctly stated, "When the number of objects to be counted exceeds the span of apprehension, a person apparently cannot attend to all of the objects at once and simultaneously count them" (p. 15). We have shown that, if one controls for selective eye movements, span of apprehension cannot be increased with longer exposure to the stimuli. Beyond 4 objects, subjects must count the targets serially, and in our experiments they required eye movements to be able to do this without error.
The fourth point is that selective eye movements are apparently also required to effectively exploit configural cues that make larger numerosities easier to enumerate. A number of studies have shown the facilitative effects on enumeration, in general, of patterns that can be subjectively broken into subgroups by the perceiver (e.g., Atkinson, Francis, \& Campbell, 1976; Frick, 1987; Shrager et al., 1982; van Oeffelen \& Vos, 1982). However, the present results show that when eye movements could not be employed to mediate that process, adding large amounts of extra inspection time did not increase, beyond the standard subitizing range, the number of objects that could be enumerated with perfect accuracy. Presumably, the function of selective eye movements under these circumstances is to first pick out which items are to be considered as part of which groups and then to mark off which groups have been processed. This is particularly interesting since the subjects in Experiment 2 could clearly make out the distinct subgroups in the stimuli and even reported higher confidence in their enumerations. Nevertheless, their performance was no better under such conditions than when the stimuli were linearly configured.

To reiterate, our results suggest that covert attention enables up to 4 items to be identified and individuated sufficiently accurately to enable enumeration to proceed without error. This is converging evidence for Trick and Pylyshyn's theory of subitizing as a phenomenon associated with the mechanisms of the attention system. What is less clear is the reason for occasional failures in enumeration beyond the subitizing range, as found in our afterimage conditions. Given that our subjects could count, the basis for these failures must lie in the individuation component of enumeration.

To avoid recounting, whether in subitizing or in the counting of larger arrays, items to be enumerated must be individuated by being identified and each somehow marked. This also helps to identify the remaining uncounted items. Apparently, there are tagging mechanisms that can be applied flawlessly to small (or uncomplicated) collections of objects under all conditions, perhaps such as FINSTs (Trick \& Pylyshyn, 1993, 1994) or "object files" (Kahneman, Treisman, \& Gibbs, 1992). For larger collections, these mechanisms work well for normal visual images when objects are individuated one at a time, but seem not to be so applicable to afterimages. Our results suggest that this is because such serial tagging requires the use of the oculomotor system, or "pointing" with the eyes. This method is not applicable to afterimages because eye movements will not pick out different parts of the image. The image resides only on the subject's retina and not in real physical space; it simply moves in toto with the eyes.

It is possible that the mechanism that underlies accurate pointing with the eyes is the phenomenon known as inhibition of return (IOR) (e.g., Posner \& Cohen, 1984). This mechanism would seem to be the perfect one to exploit for visual enumeration as it suppresses reattending 
to spatial locations and predictably moving objects (e.g., Tipper, Weaver, Jerreat, \& Burak, 1994) that have been recently examined - something to be avoided during accurate counting. Furthermore, Rafal, Calabrisi, Brennan, and Sciolto (1989) showed that subjects must at least prepare to make a saccade for IOR to occur. This means that it should be seen only in the overt attentional component of enumeration. Presumably such a mechanism would not apply to percepts resulting from afterimages, because they neither occupy real spatial coordinates nor move along predictable paths. Thus it may be that IOR plays some role in accounting for why our subjects, in enumerating more than 4 dots, fared less well with afterimages than with standard visual input. However, some controversy exists over the real characteristics of IOR (e.g., Wolfe \& Pokorny, 1990), and so this link must remain speculative at present.

One other possible accounting for the decrease of accuracy with increasing numerosity suggests that serial enumeration is a process whereby each step produces a result to within a few percent of perfect accuracy. ${ }^{1}$ As numerosity increases, the error term would mount until it represented the $20 \%-30 \%$ magnitudes seen in our subjects' postsubitizing performance. However, there are a few reasons to believe that such an account does not explain the data collected in our experiments. One reason is our finding that, in Experiment 1 at least, there was an extremely small probability that the lack of errors seen in the subitizing range resulted from the same process that was responsible for the postsubitizing errors. Another reason is that any simple serial model of the type outlined above would be hard pressed to explain the discontinuities seen in subitizing data. This may not be a problem because, as Frick (1987) points out, the intercepts for postsubitizing enumeration are usually negative. This suggests that up to 4 items are subitized and counting begins with numerosities only beyond that range. This, of course, means that the error term for counting would have to start at around $20 \%$ and then should reach around $80 \%$ by the time 8 items were being counted, which was not the case.

So, at present, we do not have a clear explanation for the appearance of errors beyond the subitizing range in the afterimage conditions of Experiments 1 and 2 and in Experiment 3 . We know that eye movements could not be used to selectively individuate items in these conditions. This left the subjects with only the mechanisms of covert attention to be used for the task of object individuation. What remains to be explained is why such processes were prone to occasional error.

These data lead to one further speculation about the relationship between subitizing and attention. They suggest that the elusive explanation of the limit of the subitizing process may be that it is simply the same as the limit of attentional pickup. Our results and those of Atkinson, Campbell, and Francis (1976) show that, when eye movements are rendered ineffective, accurate indi- viduation of items is limited to around 4 objects. Trick and Pylyshyn (1993) showed that when attentive search is induced for targets whose numerosity is within the subitizing range but whose similarity to distractors requires item-by-item individuation, the characteristic subitizing profile disappears. Therefore, it appears that the number of objects that can be subitized may be a function of the limit of the attentional pickup capacity for a given type of array.

Evidently, much more still needs to be done in order to fully understand the information-processing basis of visual enumeration processing. However, our findings that the subitizing limitation is independent of inspection time and stimulus configuration should help to narrow the search for that explanation.

\section{REFERENCES}

Atkinsón, J., Camperl.l, F. W., \& Francis, M. R. (1976). The magic number 4 plus or minus $0:$ A new look at visual numerosity judgements. Perception, 5, 327-334.

Atkinson, J., Francis, M. R., \& Campbell, F. W. (1976). The dependence of the visual numerosity limit on orientation, colour, and grouping in the stimulus. Perception, 5, 335-342.

DehaEne, S., \& Cohen, L. (1994). Dissociable mechanisms of subitizing and counting: Neuropsychological evidence from simaltanagnosic patients. Journal of Experimental Psychology: Human Perception \& Performance, 20, 958-975.

ERIKSEN, C. W., \& ST. JAMES, J. D. (1986). Visual attention within and around the field of focal attention: A zoom lens model. Perception \& Psychophysics, 40, 225-240.

FRICK, R. W. (1987). The homogeneity effect in counting. Perception \& Psychophysics, 41, 8-16.

Gelman, R., \& Gallistel, C. R. (1978). The child's understanding of number. Cambridge, MA: Harvard University Press.

Gerrits, H. J. M., van Erning, L. J. Th. O., \& Eijkman, E. G. K. (1988). Afterimages: A collective term for percepts of different origins. Experimental Brain Research, 72, 279-286.

HoYer, W. J. (1990). Levels of knowledge utilization in visual information processing. In T. H. Hess (Ed.), Aging and cognition: Knowledge organization and utility (pp. 389-409). Amsterdam: Elsevier, North-Holland.

Kahneman, D., Treisman, A., \& GibBs, B. J. (1992). The reviewing of object files: Object-specific integration of information. Cognitive Psychology, 24, 175-219.

LABERge, D. (1983). Spatial extent of attention to letters and words. Journal of Experimental Psychology: Human Perception \& Performance, 9, 371-379.

Mandler, G., \& Shebo, B. J. (1982). Subitizing: An analysis of its component processes. Journal of Experimental Psychology: General, $111,1-22$.

Posner, M. I., \& Cohen, Y. A. (1984). Components of visual orienting. In H. Bouma \& D. G. Bouwhuis (Eds.), Attention and performance $X$ (pp. 531-556). Hillsdale, NJ: Erlbaum.

Rafal, R. D., Calabrisi, P. A., Brennan, C. W., \& Sciolto, T. K. (1989). Saccade preparation inhibits reorienting to recently attended locations. Journal of Experimental Psychology' Human Perception \& Performance, 15, 673-685.

Shrager, J., Klahr, D., \& Chase, W. G. (1982, November). Segmentation and quantification of random dot patterns. Paper presented at the 23rd Annual Meeting of the Psychonomic Society, Minneapolis.

SVEnson, O., \& SJöberG, K. (1983). Speeds of subitizing and counting processes in different age groups. Journal of Genetic Psychology, 142, 203-211.

Tipper, S. P., Weaver, B., Jerreat, L. M., \& Burak, A. L. (1994). Object- 
based and environment-based inhibition of return of visual attention. Journal of Experimental Psychology: Human Perception \& Performance, 19, 478-499.

Townsend, J. T. (1990). Serial vs. parallel processing: Sometimes they look like Tweedledum and Tweedledee but they can (and should) be distinguished. Psychological Science, 1, 46-54.

Trick, L. M., \& Pylyshyn, Z. W. (1993). What enumeration studies can show us about spatial attention. Evidence for limited capacity preattentive processing. Journal of Experimental Psychology: Human Perception \& Performance, 19, 331-351.

Trick, L. M., \& Pylyshyn, Z. W. (1994). Why are small and large numbers enumerated differently? A limited-capacity preattentive stage in vision. Psychological Review, 101, 80-102. van Oeffelen, M. P., \& Vos, P. G. (1982). Configural effects on the enumeration of dots: Counting by groups. Memory \& Cognition, 10, 396-404

Wolfe, J. M., \& POKORNY, C. W. (1990). Inhibitory tagging in visual search: A failure to replicate. Perception \& Psychophysics, 48, 357362.

\section{NOTE}

1. We would like to thank one of the anonymous reviewers for suggesting the issues discussed in this alternative interpretation.

(Manuscript received June 12, 1995;

revision accepted for publication November 27, 1995.) 